Influence of Hunger on Olfactory Acuity

A HUNGRY animal is considered to be in a state of high nervous irritability leading to a heightened level of sensory and motor excitation. As glycogen stores are depleted, blood glucose-levels decrease, circulating epinephrine rises and stimulates the reticular formation of the brain, which in turn produces cortical arousal and facilitation of motor and sensory activity, lowering the threshold for all external sensory stimuli1. A satiated animal, by contrast, is relaxed, disinterested in the outside world, sleepy, and consequently should have a high sensory threshold.

There are conflicting opinions on whether olfactory acuity is a reliable, reproducible index of appetite, hunger, or satioty. According to Goetzl ${ }^{2}$, freely selected meals, as well as administration of sucrose solutions, were preceded by increased olfactory acuity and followed by decreased acuity. Janowitz and Grossman ${ }^{3}$ were unable to duplicate Goetzl's results. Subsequent investigators, however, have reported that olfactory acuity was higher before lunch than after lunch ${ }^{4}$. Coffe日 and citral, respectively, were used by the first two investigators, whereas isoamylacetate, $n$-butanol, and oil of cloves were tested by the third. Their respective testing methods were the Elsberg blast-injection technique, sniffing odour-saturated air in an olfactorium, and sniffing from a series of testtubes.

An olfactometer designed to deliver calculated amounts of odour in purified air, at controlled flow rates, temperatures, and humidities, and utilizing natural breathing, was used ${ }^{5}$. Experimental subjects consisted of University personnel, 11 males and 1 female, 30-58 years of age, selected on the basis of their proved olfactory acuity and reproducibility of judgment. Citral was found to deteriorate rapidly, changing its odorous characteristics, therefore 2-heptanone, a highly stable compound which smells like mild blue cheese, was substituted. The panel was divided into two groups which were tested alternately just before lunch (11 a.m.-12.15 p.m.) and just after lunch (1 p.m.--2.15 p.m.).

Table 1. Olfactory Responses preceding and following LUNoh Subject Sex No. of judgınents Correct response (per cent)

$$
\begin{aligned}
& A \\
& B \\
& C \\
& D \\
& E \\
& F \\
& G \\
& G \\
& B \\
& I \\
& J \\
& K \\
& L
\end{aligned}
$$

Average

$\begin{array}{cr}\text { No. of jud } & \text { p.m. } \\ \text { a.m. } & 480 \\ 400 & 480 \\ 370 & 350 \\ 400 & 490 \\ 290 & 420 \\ 380 & 380 \\ 330 & 490 \\ 290 & 190 \\ 450 & 420 \\ 270 & 260 \\ 350 & 490 \\ 450 & 470 \\ 450 & 440 \\ 369 & 407\end{array}$

$$
\begin{aligned}
& a .1 \\
& 65 \\
& 62 \\
& 71 \\
& 69 \\
& 66 \\
& 77 \\
& 69 \\
& 72 \\
& 65 \\
& 70 \\
& 68 \\
& 77 \\
& 68
\end{aligned}
$$

significant at the $0 \cdot 1$ per cent level of probability. Thus, using an olfactometer-presentation of 2-heptanone by a constant stimulus methodology, olfactory acuity was higher after lunch than before.
H. W. Berg
R. M. PANGBORN
E. B. ROESSLER
A. D. WEBB

College of Agriculture,
University of California, Davis.

'Dell, P. C., Neurological Basis of Behavior, 187 (Ciba Symposium, 1958; ${ }^{2}$ Goetzl, F. R., and Stone, F., Gastroenterol., 9, 444 (1947). Goetzl, P. R. Goldschmidt, M.. Wheeler, P., and Stone, F., ibid., 12, 252 (1949). Goetzl, F. R., Abel, M. S., and Ahokas, A. J., J. App. Physiol., 2, 553 (1950). Goetzl, F. R., Ahokas, A. J., and Goldschmidt, M., ibid., 4, 30 (1951).

3 Janowitz, H. D., and Grossman, M. I., J. App. Physiol., 2, 217 (1949).

4 Hammer, F. J., J. Comp. and Physiol. Psychol., 44, 403 (1951). Schneider, R. A., and Wolf, S., J. A pp. Physiol, 8, 337 (1955). Fruchtgott, E. "Ough, C. S., and Stone, H., J. Foor Sei., 26, 452 (1961).

\section{A Vertical-Horizontal Illusion for Movement perceived tactually}

THERE is a well-known visual illusion in which a vertical line is judged as longer than a horizontal line of the same length. The illusion also holds, though attenuated, when lines are perceived tactually, even in blind subjects ${ }^{1}$. This communication is concerned with a similar error which is made when speed of movement is assessed tactually, but which does not show when the judgment is made visually.

The apparatus consisted of two 6-in. drums the speed of rotation of which could be varied. One was mounted with its axis vertical, the other horizontal. The drums were covered with paper embossed by means of Braille writing equipment with a pattern of dots in triplets, $1 \mathrm{~mm}$ across, and with an interval of $12 \mathrm{~mm}$ between triplets of dots. In both experiments the subject rested his finger-tips against the dots on the surfaces of the drums as they rotated. Thus one hand was held in a vertical plane and the other horizontally.

In the first experiment, subjects were required to adjust the speed of one drum so that it appeared tactually (with eyes shut) to rotate at the speed of the other. All 12 subjects adjusted the speed so that the drum with vertical axis was objectively rotating faster. This means that the speed of the dots moving vertically past the fingers was overestimated.

The finding was tested in another way. The two drums were rotated at the same speed with dots passing the fingers at $27.5 \mathrm{~mm} / \mathrm{sec}$, and subjects were each required to make a single tactile judgment as to which drum was rotating faster (subjects were not allowed to say 'equal'). Of 24 subjects, 19 reported that the drum with horizontal axis (vertical movement) was the faster. On a $\chi^{2}$ test this result is expected in less than 1 per cent of cases by chance. Another 24 subjects made the judgment visually. Thirteen reported the drum with horizontal axis as faster, 11 the drum with vertical axis. In the tactile judgments the effect did not depend on whether the dots were moving away from or towards the body, nor did it depend on handedness.

The results suggest a vertical-horizontal asymmetry in tactile perception. The most plausible explanations of visual illusions ${ }^{2}$ are in terms of eye-movements, or configurational effects or depth perception. None of these appears to explain the present illusion.

D. LIDDLE

B. M. Foss

Department of Psychology,

Birkbeck College,

University of London.

beforo lunch, subjects $C$ and $H$ showed no difference, whereas the remaining nine were more acute after than before lunch. The $t$-test for variance indicated the difference between morning and afternoon panels was
1 Hatwell, Y., Anné Psychol., No. 1, 11 (1960).

Boring, E. G., Sensation and Perception in the History of Experimental Psychology, 244 (1942). 